OOMPUTATIONAL MCATHEMATICS

BANACH OENTER PUBLICATIONS, FOLUMT 13

PWN - POLISH SOIENTIFIO PUBLISHERS

WARSAW 1984

\title{
КОНСТРУИРОВАНИЕ РАЗНОСТНЫХ СХЕМ С ЗАДАННЫМИ СВОЙСТВАМИ
}

\author{
ІОР РИ ШО КИ Н \\ Вияислительиый Центр СО АН СССР, \\ Iірастолрск, CCCP
}

Практика решения задач прикладной механики показывает, что бев создания новых эффективных конечно-разностных алгоритмов, а также методов аналива разностных схем, нельзя получить репение многих глассов важных прикладных задач. Появление и практическое использонание большого количества разностных схем привело $\mathrm{k}$ повышению роли критериев отбора и сравнения схем.

Одним из эффективных методов построения разностных схем с заданными свойствами является метод диффферепциального приближения, нашедший в настоящее время широкое применение. Әфффентивность метода подтверждена обширной практикой. Возможность использования дифференциальных приближсний при исследовании раэностных схем впервые была угазана в 50-х годах А. И. Жуковым для случая простейшего уравнения переноса с постоянными коэффициентами. В 1968 году в работах автора и Н.Н. Яненко было сформулировано понятие первого дифференциального приближения (п.д.п.) для произвольной разностной схемы с постоянными коэффициентами и доказаны теоремы о связи устойqивости простьх и мажорантных схем и корректности их п.д.П. для гиперболической системы дифференциальных уравнений первого порядна. Впоследствии идея использования диффференциальных приближений при анализе свойств схем получила широное развитие в исследованиях автора и $\mathrm{H}$. Н. Яненко, а такне в исследования других советских и эарубенных математиков. Метод дифференциального приближения позволяет строить новые 
разностные схемы с заранее определенными свойствами, проводить анализ разностных схем, сравнцвать их менду собой, а таняе длассифицировать их по определенным признанам.

Јитература по методу дифферснцилыного приблияения насчнтывает в настояцее время свыше 170 наименований. Большинство реаультатов этих исследований носит эврнстический хараптер, и правильность их подтверждена многочислеными расчетами. Теоремы о свяэи устойчивости разностных схем п свойств п.д.І. в случае гиперболических систем уравнений доказаны автором и I- H. Яненко. Устойчивость разностных схем исследуется методом цифференциального приблияения в работах автора, H. I-I. Яненго и их учепнков, Н. Н. Апугиной, О. М'. Белоцерговсгого и IО. М. Давыдова, 'Т. Харлоу, Ц. Хирта п др. Мегод диффферепцильпого приблинения огазался весьма полезпым ири анализе цисперсиониых свойств разностных схем. Особенно это видио при исследовании свонств разностных схем методом типа „частиц в ячейке" в работах I- Н. Анучнной, В. li. IГетренко, Н. I. Яненго и автора, О. М. Јстоцсрновстого, IО. М. Мавыдова и их угеников, Т. Харлоу и сго ученитов. ІТа основе диг)(ре-

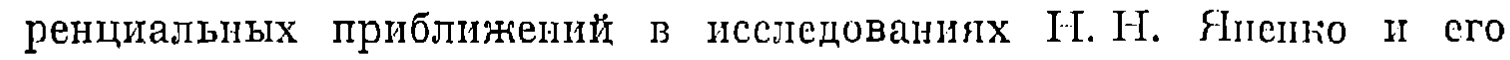
ученшков раэвита методнка днфференциального анализатора, нашедшего применение при лонализации скапнов п разностиых решениях. Автором, Н. Н. Яненио и А. И. Урусовым прсдлонен петод постросния схем на произвольной сеткс па оспове разностнои схемы, ғаданшой на равномерной сстке, оенованый на анализе дифференцианынх приближенпй схсм. Ан. Г. Марчуном, З.И. Федотовой и антором псследован вопрос о связи свойств п.д.п. и свойства полной лопсервативности разностных схем в смысле А. А. Самарского - IO. П. Попова. В работах автора и I-I. I- Яненіо введено понятие инвариалтных разностных схем, то есть схем, П.д.п. поторых допуснает пслоторуіо группу преобразований. Практика растетов показывает, түо непнвариантность разностных схем относительно группы преобразовани допуснаемых нсходной системой дифферснциальных уранпенй, прн-

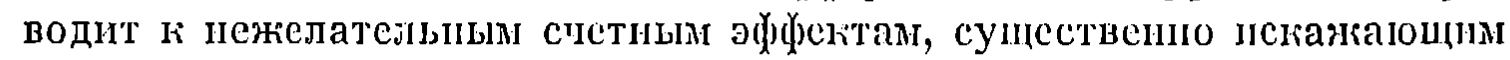

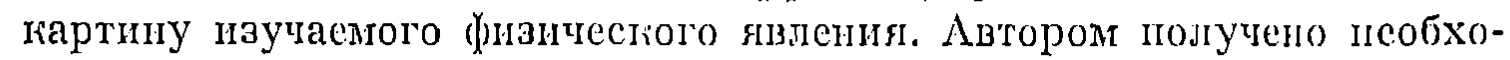

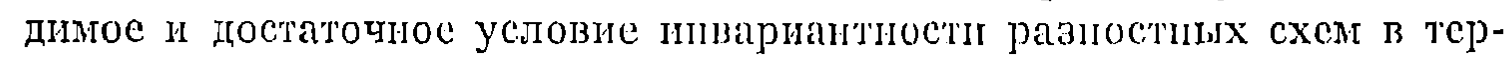

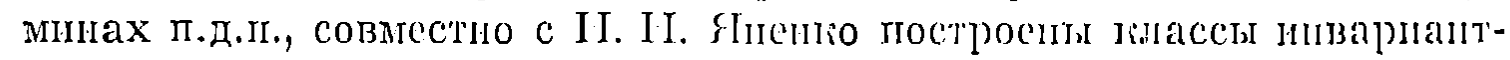

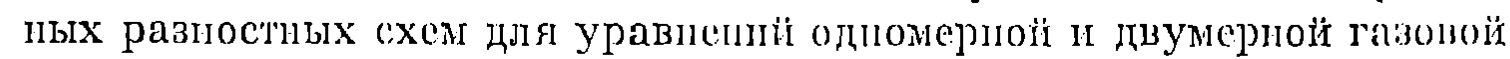
динамики, рсзулытаты расчетов то готорым привсдены в работах

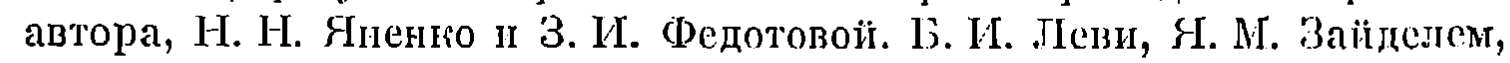

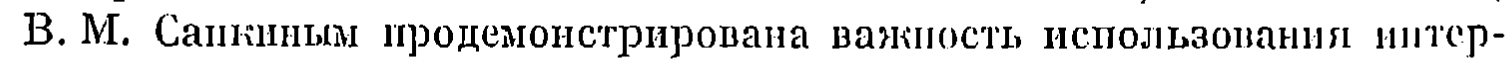
валыных разностиых схем при численом моделнровани задат ()п,-

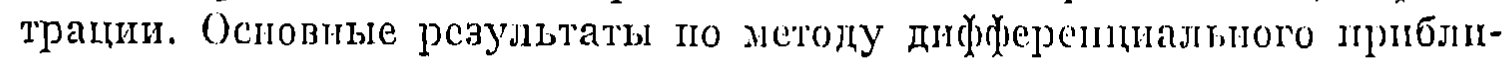
жения излонены в мопоград)ии [1]. 
2

Сформулируем понятия диффференциального представления ил дифференциального приблшженля разностных схем.

Пусть разностная схема

$$
\Lambda_{1} u^{n+1}(x)=\Lambda_{0} u^{n}(x)
$$

аппрогскмирует дифференциальное уравнение

$$
\frac{\partial u}{\partial t}=Z u
$$

Здесь оператор $\Lambda_{1}$ обратнм, $Z=Z(t, x, D), \Lambda_{k}=\Lambda_{k}(t, x, \tau, h, T)(k=$ $=0,1), D=\frac{\partial}{\partial x}=\left\{\frac{\partial}{\partial r_{1}}, \ldots, \frac{\partial}{\partial x_{s}}\right\}, T=\left\{T_{1}, \ldots, T_{s}\right\}, x=\left\{x_{1}, \ldots, x_{s}\right\} \in$ $\in R_{s}, h=\left\{h_{1}, \ldots, h_{s}\right\}, t=n \tau, \tau-\operatorname{mar}$ по оси $t, h_{j}-$ шаг по осп $x_{j}$, $T_{j}$-онератор сдвига по оси $x_{j}(j=1, \ldots, s)$.

При аппрогсимации дифферегциального уравнения разностной схемой мы совершаем переход от бесконетномерного пространства ()унццй непрерывного аргумента гі конегномерному пространству сетопых функцй п сведение уравнений для фуници тепрерывного аргумснта н алгебрапчесін соотношениям. Такое рассмотрение, удобное на праћтияе, вызывает трудности прн теоретнтеском аналиве свойств разностных схсм, так пан сеточная фунцция и функция непрерывного аргумента определены в разных пространствах. При теоретическол исследовании разностных схем зачастуг удобно рассмагривать разностные операторы в том же фунгциолальом шространстве, что и аппроксимпуеные пми дифференциальные операторы. В этом случае считало, что разностные схемы удовлетворяются функциямн непрерывного аргумента в наждой точге рассматриваемой області [2]. Мы придерживаемся именно такого подхода.

Iзвестны следугшие операторныс представления:

$$
\begin{gathered}
T_{j}=\exp \left(h_{j} D_{j}\right)=\sum_{l=0}^{\infty} \frac{h_{j}^{l}}{l !} D_{j}^{l} \quad(j=1, \ldots, s) \\
I_{0}=\exp \left(\tau D_{0}\right)=\sum_{l=0}^{\infty} \frac{\tau^{l}}{l !} D_{0}^{l}, \quad \ln \left(\exp \tau D_{0}\right)=\tau D_{0}
\end{gathered}
$$

$T_{0}$ - оператор сдвига по осп $t$. 'Гогда разностную схему (1) можно представнть в ниде

$$
e^{\tau \cdot \frac{\partial}{a t}} u=A_{1}^{-1}\left(t, x, \tau, h, e^{h D}\right) \Lambda_{0}\left(t, x, \tau, h, e^{h D}\right) u,
$$


где

$$
\exp h D=\left\{\exp h_{1} \frac{\partial}{\partial x_{1}}, \ldots, \exp h_{s} \frac{\partial}{\partial x_{s}}\right\}
$$

и, в сплу аппрокспмации, получнм

$$
\sum_{l=1}^{\infty} \frac{\tau^{l-1}}{l !} \frac{\partial^{l} u}{\partial t^{l}}=Z u+\sum_{l=1}^{\infty} \sum_{l_{1}+\ldots+l_{s}=l}^{l} a_{l_{1} \ldots l_{s}} \frac{\partial^{l} u}{\partial x_{1}^{l_{1}} \ldots \partial x_{s}^{l_{s}}}
$$

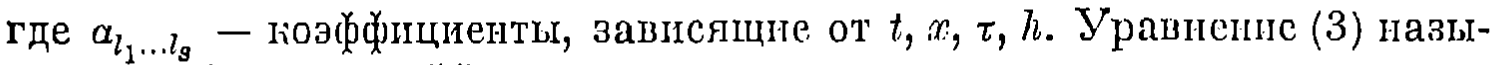
вается $T$-формої дифферепинльного представления раэностой схемы (1). Дифференциальне представленце разностной схемы чале рассматривают в другой (рорме, а именно, в $\Pi$ - қорме, готорая получается из $\Gamma$-формы заменой производных $\partial^{l} u / \partial t^{l}(l \geqslant 2)$ через производные по $x$, пспользуя саму $\Gamma$-форму ди)фференциального представления. Из уравнения (3) имесм

$$
\begin{aligned}
& \frac{\partial u}{\partial t}=\frac{1}{\tau} \ln \left\{E+\tau\left[Z+\sum_{l=1}^{\infty} \sum_{l_{1}+\ldots+l_{s}=1}^{l} a_{l_{1} \ldots l_{s}} \frac{\partial^{l} u}{\partial x_{1}^{l_{1}} \ldots \partial x_{s}^{l_{s}}}\right]\right\} u= \\
& =Z l_{l}+\sum_{l=1}^{\infty} \sum_{l_{1}+\ldots+l_{s}=1}^{l} c_{l_{1} \ldots l_{s}} \frac{\partial^{l} u^{l}}{\partial x_{1}^{l} \ldots \partial x_{s}^{l}},
\end{aligned}
$$

где $c_{l_{1} \ldots l_{s}}$ - ноэффициенты, завислшие от $t, x, \tau, h, E$ - тондественный оператор. Уравнение

$$
\frac{\partial u}{\partial t}=Z u+\sum_{l=1}^{\infty} \sum_{l_{1}+\ldots+s_{s}=1}^{l} c_{l_{1} \ldots l} \frac{\partial^{l}{ }^{\prime} l}{\partial x_{1}^{l} \ldots \partial x_{s}^{l}}
$$

называется $\Pi$-формой диф)ференциального ггедставления разпостной схемы (1).

Указанный формальный способ построения $\Gamma$-формы и $\Pi$-рорормы цифсыеренциального представления разностной схемы на прагтне можно вылолнить стедуоцци образом. Сделасм это па примерс разпостиой схемы

$$
u^{n+1}(x)=\Lambda\left(t, x, \tau, h, T_{0}, T\right) u^{n}(x),
$$

аппронсимирующей уравнение (2) с порядком $\gamma$, причсм, предполгони, что $h=h(\tau)$. В общем случае процесс выполнястся аналогично. Разэган в разностной схеме (5) фунцци вица $u^{n+a}\left(x_{1}+\beta_{1} h_{1}, \ldots, x_{s}+\beta_{s} h_{s}\right)$, где $\alpha, \beta_{1}, \ldots, \beta_{s}$ - некоторые числа, в ряд по параметрам $\tau, h_{j}$, по- 
лучим

$$
\frac{\partial u}{\partial t}=\bar{Z}\left(t, x, \tau, h, D_{0}, D\right) u=Z^{1}\left(t, x, \tau, h, D_{0}, D\right) u+R
$$

где

$$
\begin{gathered}
Z^{1} u=Z u+\tau P_{1}\left(t, x, D_{0}, D\right) u+\ldots+\tau^{\gamma} P_{\gamma}\left(t, x, D_{0}, D\right) u, \\
R=\sum_{l>\gamma} \tau^{l} P_{l}\left(t, x, D_{0}, D\right) u .
\end{gathered}
$$

Уравнение (6) есть $\Gamma$-форма дифференциального представления разностноң схемы (5). П-форма дифференциального представления

$$
\frac{\partial u}{\partial t}=\tilde{Z}(t, x, \tau, h, D) u
$$

разностиой схемы (5) получается из $\Gamma$-формы (6) заменой в правоц части уравненія (6) пронзводных по $t$ и смешанных пронзводных по $t$ и $x$ пронзводными по $x$, используя $\Gamma$-форму диффференциального представления.

В работе [1] дан алгоритм получения $\Pi$-формы дифференциального представления. Для разностных схем, используемых на праптиліс при аппроксимации уравнения переноса с постоянными коэффйциентами, даны регуррентные формулы для лоэффффициентов $\Pi$-формы дифференцлалього представления.

Предположим, тто разностная схема (1) имеет порядкі аппроксимации $\gamma_{1}$ II $\gamma_{2}$ по $t$ и $x$ соответсгвенно. Тогда, отбрасывая в уравнснин

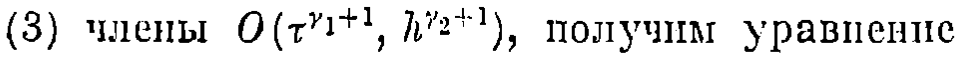

$$
\frac{\partial u}{\partial t}+\frac{\tau}{2} \frac{\partial^{2} u}{\partial t^{2}}+\ldots+\frac{\tau^{\gamma_{1}}}{\left(\gamma_{1}+1\right) !} \frac{\partial^{\gamma_{1}+1} u}{\partial t^{\gamma_{1}+1}}=Z u+Z_{1}(D) u,
$$

где $Z_{1}(D)$ - неноторый дифференциалыный оператор, коэффициенты ноторого пімет порндкін $O\left(\tau, \ldots, \tau^{\gamma_{1}}, h, \ldots, h^{\prime \prime 2}\right)$, и ко'орое называется $\Gamma$-формой первого дн()еренциального прнблияения (п.д.п.) разностпой схемы (1). Если в $I$-формс дифферепциального представления (4)

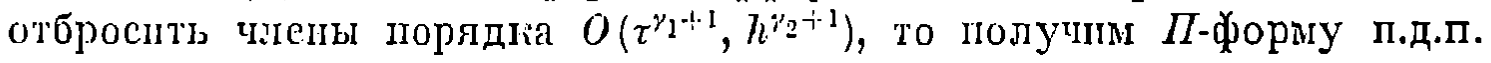

$$
\frac{\partial u}{\partial t}=Z u+\tilde{Z}_{1}(D) u \text {. }
$$

Здесь $\tilde{Z}_{1}(D)$ - лскоторый дифференциальный оператор, коэффицциенты поторого пмелот порядон $O\left(\tau^{\gamma_{1}}, h^{\gamma_{2}}\right)$. В случае разностной схемы $(\tilde{5})$ $\Gamma$-форма п.д.п. имеет нид $\hat{c} u / \partial t=Z^{1} u$, а $\Pi$-форма п.д.п. $\partial u / \partial t=$ $=Z u+\tau^{\prime} P_{\gamma}(t, x, D) \imath$. Нетрудно впдегь, что $\Pi$-форма П.д.П. (8) разностной 
схемы (1) получается пз $\Gamma$-формы п.д.п. (7) в силу аппронсимации формальной заменой пронзводных по $t$ до порядіа $\gamma_{1}$ через производные по $x$, используя исходнос дифферешцильпе уравнспне.

Огбрасывая в уравнении (4) члены порядка $O\left(\tau^{\gamma_{1}+2}, h^{\gamma_{2}+2}\right), O\left(\tau^{\gamma_{1}+3}\right.$, $h^{\gamma_{2}+3}$ ) Iи т.д., получим соответственно $\Pi$-рормы второго, трстьего й т.д. дифференцилльных приблияений. Аналогично определяотсл $\Gamma$-формы второго, третьсго и последугщих дифференциалыых приблиясний.

В дальнейшем, где не оговорено особо, под дифиеренцильным представлением і п.д.п. раэностной схемы будем попимать $\Pi$-форму дифференциального представленя и $I I$-форму п.д.п.

С.)ормулирусм определения ряда свойств разностин схем в терминах п.д.Іг. Вначале определим свойства $\Pi_{j}^{(p, \gamma)}, D_{j}^{(p, \gamma)}, P_{j}^{(\eta, \gamma)}$ раэностной схемы $p$-го порядла аппрогсимацин для гиперболической системы дифференциальных уравнений первого порядка с постоннными поэф)фицентами. Эти свойства означалот, что в $\gamma$-ом дифференциальном приближени $j$-й инварнант пе подвергается действио диссигации эа стет аппроксимационной вязгости и подвергается дисперсии и дополнительному персносу вдоль $j$-ой харалтеристики.

Рассмотрим гпперболическуг систему уравнений

$$
\frac{\partial w}{\partial t}=A \frac{\partial w}{\partial x}
$$

где $w=w(x, t)-$ вектор-фуункция с $m$ компонентами, $A-m \times m$ поостоянпая матрица, имеющая различные собственные значения. Обозначим через $X_{j}$ и $Y_{j}$ соответственно лгвый и правый собственные вегтора матрицы $A$, отвечающие собственному числу $\mu_{j}$, через $r_{j}$ - инвариан', переносящийся без изменения вдоль $j$-ой хараптернстин с палылюм $d x / d t=\mu_{j}$ ш удовлетворяюший уравнению

$$
\frac{\partial r_{j}}{\partial t}=\mu_{j} \frac{\partial r_{j}}{\partial x}
$$

В общем случае п.д.п. разностной схемы порядка аппронсимации $p$ आMee' вид

$$
\frac{\partial w}{\partial t}=A \frac{\partial w}{\partial x}+C_{r}^{(p)} \frac{\partial^{r} w}{\partial x^{r}}+\ldots+C_{1}^{(p)} \frac{\partial w}{\partial x}
$$

где $r$ - натуральное число, $O_{u}^{(p)}=O\left(h^{p}\right)(\alpha=1, \ldots, r)$.

Oпредельние 1. Разиостнал схема обладает свойством $\Pi_{j}^{(1), 1)}$ uли, что то эчсе, свойством $K$ вдоль j-ой xарактеристики в п.д.n. (npuнадлеэсит классу $\left.K_{j}^{(p, 1)}\right)$, если $X_{j} C_{\beta}^{(\mu)}=0(\beta=2,4, \ldots, 2[\vartheta / 2])$. 
Если $C_{\beta}^{(p)}=0$, то будем рассматривать второе д.П. разностної схемы и говорить соответственно о свойстве $\Pi_{j}^{(2,2)}$. Аналогично можно ввести свойство $\boldsymbol{K}_{j}^{(p, \gamma)}$.

В дальнейшем мы будем опускать индексы и писать просто $\boldsymbol{K}$ (в частности, в случае газовой динамики, связывая это свойство с инвариантом - энтропией).

Определение 2. Разностнцая схема обладает сильным сөойством $K_{j}$ (сильным свойством $\mathbb{K}$ вдоль $j$-ой характеристики), если $X_{j} G=e^{i \kappa \mu_{j}} X_{j}$, где $G$ - матрцца перехода разностной схемы.

Оптеделенде 3. Разностная схема обладает свойством $D_{j}^{(p, 1)}$ или, что то же, свойством $D$ (дисперсии) вдоль $j$-ой характеристики в п.д.n. (принадлежит классу $D_{j}^{(p, 1)}$ ) если по крайней мере одна из величин $X_{j} C_{\beta}^{(p)} Y_{j} \neq 0\left(\beta=3,5, \ldots, 2\left[\left(\gamma^{*}+1\right) / 2\right]-1\right)$.

Аналогично определяется свойство $D_{j}^{(p, \gamma)}$, если в дифференциальных приближениях до $(\gamma-1)$-го порядка включительно отсутствугт все величины с неqетными производными, начиная с третьей производной, а в дифференциальном приближении порядка $\gamma$ есть по крайней мере один член с нечетной производной порядка не меньше третьего.

ОпРядтлене 4. Разностная схема $p$-го порядка аппроксимачии обладает свойством $P_{j}^{(p, 1)}$ или, что то же, переноса вдоль j-ой характеристики в п.д.n. (принадлежсит классу $\left.P_{j}^{(p, 1)}\right)$, если $X_{j} C_{1}^{(p)} Y_{j} \neq 0$. $\Pi$ Пи этом вдоль $j$-ой характеристики происходит перенос с опережсением (свойство $\left.P_{j+}^{(p, 1)}\right)$, если $X_{j} C_{1}^{(p)} Y_{j}<0$ и с запаздыванием (свойство $P_{j-}^{(p, 1)}$ ), ecлu $\bar{X}_{j} C_{1}^{(p)} Y_{j}>0$.

В случае, когда в дифференциальных приближенилх разностной схемы до $(\gamma-1)$-го порядка включительно отсутствугт члены вида $C_{1}^{(p)} \frac{\partial w}{\partial x}$, а в диффферснциальном приближении порядка $\gamma$ тагой член присутствует, будем говорить о свойстве $P_{j}^{(p, \gamma)}$ (класс $P_{j}^{(p, \gamma)}$ ).

Замстим, что если разностная схема принадлежит классу $\Pi_{j}^{(p, 1)}$ и не принадлежит нлассу $D_{j}^{(p, 1)} \cap P_{j}^{(p, 1)}$, то соответствующий инвариант $r_{j}$ в П.д.п. переносптся без изменения вдоль $j$-ой характеристики с наклоном $\mu_{j}$ и, следовательно, удовлетворяет в п.д.п. уравненио (9).

ОПределение 5. $B$ случае системы уравнений газовой динамики разностнал схема обладает свойством $M$ (принадлежсит классу $M$ ), если в ее п.д.п. выполнен закон сохранения массы.

Определение 6. В случае системы уравнений әазовой динамики разностная схема обладает свойством $\vec{K}$, если в п.д.п. энтропия сохраняется. 
ОпРвделтние 7. В случае системы уравнений газовой динамики разностнал схема обладает свойством $Г$ (принадлезсит классу $\Gamma$ ), если ее п.о.п. инвариантно относительно преобразования Галилея.

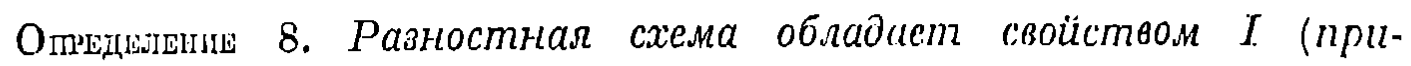

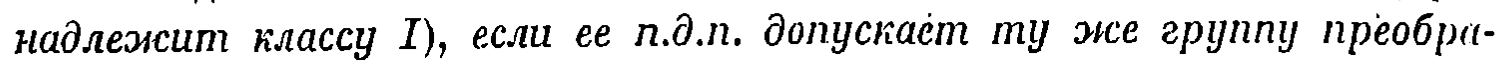
әований, что и исходнал система диябберенциальных уравнений.

В таблице 1 приведены п.д.п. разностных схем для уравпения

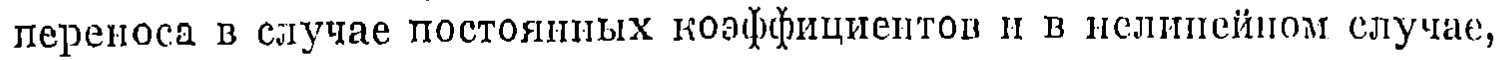
а также даны сведения о гонсервативностн и нпрариантиости относительно сдвигов по $t, x$ и преобразованиї Ганинея и растинсния.

В таблице 2 показаны п.д.п. ряда разностних схсм лич у'равиений газовой динамики как в эйлеровых, так и в лаграпненых лоординатах,

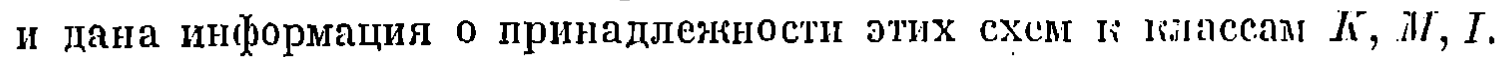

В таблице 3 содержатся сведения о разпостних схемах дыя уравнений газовой динамини в недивергентпой форме в лаграпясынх гоординатах.

Таблица 1

\begin{tabular}{|c|c|c|}
\hline No & Схема & ПГ. т̆.п. для уравнөнил $L u=u_{l}+a u_{x}=0$ \\
\hline 1 & Пакса & $L u=\frac{h^{2}}{2 \tau}\left(\mathrm{L}-\varkappa^{2} a^{2}\right) u_{x x}$ \\
\hline 2 & Годунова & $L u=\frac{h}{2}\left(a \operatorname{sign} a-x a^{2}\right) u_{x x}$ \\
\hline 3 & Pycamona & $L u=\frac{h}{2}\left(w a \operatorname{sign} a-x a^{2}\right) u_{x x}$ \\
\hline 4 & Иңвариантнал & $L u=\frac{h^{2}}{2 \tau} a u_{x x}$ \\
\hline 5 & Лакса-Вендроффа & $L u=\frac{h^{3}}{B \tau}\left(x^{3} a^{3}-x a\right) u_{x x x}$ \\
\hline 6 & $\begin{array}{l}\text { Hefimafi с цен- } \\
\text { тральной аппропсы- } \\
\text { мацпеi }\end{array}$ & $L \iota=\left\{\begin{array}{l}\frac{\tau}{2}(2 \gamma-1) a^{2} u_{x x}, \gamma+\frac{1}{2} \\
-\frac{h^{2}}{i} a\left(2+\star^{2} a^{2}\right) u_{x x x}, \gamma=\frac{1}{2}\end{array}\right.$ \\
\hline 7 & 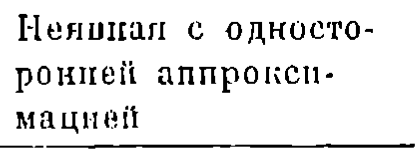 & $L u=\frac{h a}{2}[(2 y-1) x a+\operatorname{sign} a] u_{x x}$ \\
\hline 8 & $\begin{array}{l}\text { Предиктор-горрегтор } \\
\text { с центральными } \\
\text { раэностнми } \\
\text { D преди іторе }\end{array}$ & $L u=\left\{\begin{array}{l}\frac{\tau}{2}(2 \gamma-1) a^{2} u_{x x}, \gamma \neq \frac{1}{2} \\
-\frac{h^{2}}{6} a\left(x^{2} a^{2}+2\right) u_{x x x x}, \gamma=\frac{1}{2}\end{array}\right.$ \\
\hline
\end{tabular}


Продолжение табл. 1

\begin{tabular}{|c|c|c|}
\hline 9 & $\begin{array}{l}\text { Прединтор-корректор } \\
\text { с односторогними } \\
\text { равностями в пре- } \\
\text { дикторе }\end{array}$ & $L u=\left\{\begin{array}{l}\frac{\tau}{2}(2 \gamma-1) a^{2} u_{i x}, \gamma \neq \frac{1}{2} \\
-\frac{\tau h}{4}(\operatorname{sign} a) a^{2} u_{x x x}-\frac{\tau^{2}}{12} a^{3} u_{x x x}-\frac{h^{2}}{6} a u_{x x x}, \\
\gamma=\frac{1}{2}\end{array}\right.$ \\
\hline 10 & $\begin{array}{l}\text { Двухпараметритесіое } \\
\text { семенство } \mathscr{L}_{\beta, 0}^{a}\end{array}$ & $L u=\frac{h^{3}}{6 \tau}\left(\varkappa^{3} a^{3}-x a\right) u_{x x x}$ \\
\hline 11 & $\begin{array}{l}\text { Двухпараметритесіое } \\
\text { семенстьо } \mathscr{L}_{0, \varepsilon}^{\text {a }}\end{array}$ & $L u=\frac{h^{3}}{6 \tau}\left(\varkappa^{3} a^{3}-\not a\right) u_{x x x}$ \\
\hline 12 & "leap-frog" & $L u=\frac{h^{3}}{6 \tau}\left(x^{\mathrm{J}} a^{3}-\varkappa a\right) u_{x \tau x}$ \\
\hline 13 & Итерационыные схемы & $L u=\theta \pi a^{2} u_{x x}$ \\
\hline 14 & $\begin{array}{l}\text { Двухпараметритестое } \\
\text { семейстро } \mathscr{L}_{1 / 2,0}^{a, \omega} \\
\end{array}$ & $L u=\frac{1}{6} \tau l^{2} a^{2}\left(1-\frac{x^{2} a^{2}}{4}\right) u_{x x x x}-\omega \frac{h^{4}}{24 \tau} u_{x x x x}$ \\
\hline 15 & $\begin{array}{l}\text { Трехпараметрическое } \\
\text { семейство } \mathscr{L}_{0, \boldsymbol{c}}^{a, \omega} \\
\end{array}$ & $L u=\frac{1}{6} \tau h^{2} a^{2}\left(1-\frac{x^{2} a^{2}}{4}\right) u_{x x x x}-\omega \frac{h^{4}}{24 \tau} u_{x x x x}$ \\
\hline 16 & $\begin{array}{l}\text { Балакина третьего } \\
\text { порядка аппрогси- } \\
\text { мации }\end{array}$ & $L u=\frac{5}{48} \tau h^{2} a^{2} u_{x x i c x}-\frac{\tau^{3}}{24} a^{4} u_{x x x x}-\frac{3 h^{4}}{128 \tau} u_{x x x x}$ \\
\hline 17 & $\begin{array}{l}\text { Балакина четпергого } \\
\text { порлдка аппролсл- } \\
\text { мациц }\end{array}$ & $L u=-\frac{h^{5}}{120 \tau} \approx a\left(1-\varkappa^{2} a^{2}\right)\left(4-\varkappa^{2} a^{2}\right) u_{x x x x x}$ \\
\hline 18 & Тушевон & $L u=\frac{h^{5}}{180 r} * a\left(1-\varkappa^{4} a^{4}\right) u_{i x x x x}$ \\
\hline
\end{tabular}

Продолжепие табл. 1

\begin{tabular}{|c|c|c|c|}
\hline No & П.д.п. для урапнения $L u=u_{l}+f_{x}=0, f=u^{2} / 2$ & $\begin{array}{l}\text { Fioн- } \\
\text { серва- } \\
\text { тнв- } \\
\text { IIос'ь }\end{array}$ & $\begin{array}{l}\text { Mида- } \\
\text { риагт- } \\
\text { ность }\end{array}$ \\
\hline 1 & $L u=\frac{h^{2}}{2 \tau}\left[\left(1-\varkappa^{2} u^{2}\right) u_{x}\right]_{x}$ & да & HeT \\
\hline 2 & $L u=\frac{h}{2}\left[\left(u \operatorname{sign} u-x u^{2}\right) u_{x}\right]_{x}$ & да & HеT \\
\hline 3 & $L u=\frac{h}{2}\left[(\omega \operatorname{sign} u-\varkappa u) u u_{x}\right]_{x}$ & xa & IIеT \\
\hline 4 & $L u=\frac{h^{2}}{2 \tau}\left(\alpha u_{x}\right)_{x}, \partial \alpha / \partial u=0$ & да & да \\
\hline
\end{tabular}


Іо. шокин

Продолжешн табл. 1

\begin{tabular}{|c|c|c|c|}
\hline 5 & $L u=\frac{\tau^{2}}{6}\left(u^{3} u_{x}\right)_{x x}-\frac{h^{2}}{12}\left(u^{2}\right)_{x x x}$ & да & IIET \\
\hline 6 & $L u=\left\{\begin{array}{l}-\frac{\tau}{2}(2 \gamma-1)\left(u^{2} u_{i c}\right)_{x}, \gamma \neq \frac{1}{2} \\
-\frac{\tau^{2}}{12}\left(u^{3} u_{x i}\right)_{x x}-\frac{h^{2}}{12}\left(u^{2}\right)_{x x i \tau}, \gamma=\frac{1}{2}\end{array}\right.$ & ла & IIET \\
\hline 7 & $L u=\frac{\tau}{2}(2 \gamma-1)\left(u^{2} u_{x}\right)_{x}+\frac{h}{4}(\operatorname{sign} u)\left(u^{2}\right)_{x x}$ & да & IICT \\
\hline 8 & $L u=\left\{\begin{array}{l}\frac{\tau}{2}(2 \gamma-1)\left(u^{2} u_{x x}\right)_{x}, \gamma \neq \frac{1}{2} \\
\begin{array}{rl}\frac{\tau^{2}}{6}\left(u^{3} u_{x}\right)_{x x}-\frac{\tau^{2}}{8}\left[u^{2}\left(\left(u^{2}\right)_{x x}+\left(u_{x}\right)^{2}\right)\right]_{x}- \\
-\frac{h^{2}}{12}\left(u^{2}\right)_{x x x}, \gamma=\frac{1}{2}\end{array}\end{array}\right.$ & да & IIET \\
\hline 9 & $L u=\left\{\begin{array}{l}\frac{\tau}{2}(2 \gamma-1)\left(u^{2} u_{x}\right)_{x}, \gamma \neq \frac{1}{2} \\
-\frac{\tau h}{4}(\operatorname{sign} u)\left(u^{2} u_{x x}\right)_{x}+\frac{\tau^{2}}{6}\left(u^{3} u_{x}\right)_{x x}- \\
-\frac{\tau^{2}}{8}\left[u^{2}\left(\left(u^{2}\right)_{x x x}+\left(u_{x}\right)^{2}\right)\right]_{x}-\frac{h^{2}}{12}\left(u^{2}\right)_{x x x}, \gamma=\frac{1}{2}\end{array}\right.$ & да & нет \\
\hline 10 & $\begin{aligned} L u= & \frac{\tau^{2}}{6}\left(u^{3} u_{x}\right)_{x x}-\frac{\tau^{2}}{4} a\left(u^{2}\left(u_{x}\right)^{2}\right)_{x}-\frac{\tau h(1-2 \beta)}{4}\left(u\left(u_{x}\right)^{2}\right)_{x}- \\
& -\frac{h^{2} \beta(\beta-1)}{4 a}\left(\left(u_{x}\right)^{2}\right)_{x}-\frac{h^{2}}{12}\left(u^{2}\right)_{x x x}\end{aligned}$ & да & нет \\
\hline 11 & $\begin{aligned} L u= & \frac{\tau^{2}}{6}\left(u^{3} u_{x}\right)_{x x}-\frac{\alpha \tau^{2}}{4}\left(u^{2}\left(u_{x}\right)^{2}\right)_{x}-\frac{h^{2}}{12}\left(u^{2}\right)_{x x x}- \\
& -\frac{\tau h}{4}(1-2 \varepsilon)\left(u\left(u_{x}\right)^{2}\right)_{x}\end{aligned}$ & да & нет \\
\hline 12 & $L u=\frac{\tau^{2}}{6^{6}}\left(u^{3} u_{x}\right)_{x x}-\frac{h^{2}}{12}\left(u^{2}\right)_{x x x}$ & да & нет \\
\hline 13 & $L u=\tau \theta\left[2 u\left(u_{x}\right)^{2}+u^{2} u_{x x}\right]$ & ла & нет \\
\hline 14 & $\begin{aligned} L u= & \frac{\tau h^{2}}{24 \alpha}\left(4 a u_{x}^{3}+(18 \alpha-1) u u_{x} u_{x x}+4 a u^{2} u_{x x x}\right)_{x}+ \\
& +\frac{\tau^{3}}{18 \alpha}\left[(3+2 \alpha) u^{3} u_{x} u_{x x}+(3+4 a) u^{2}\left(u_{x}\right)^{3}\right]_{x}- \\
& -\frac{\tau^{3}}{24}\left(u^{4} u_{x}\right)_{x x x}-\frac{\omega h^{4}}{24 \tau} u_{x x x x}\end{aligned}$ & ца & IreT \\
\hline
\end{tabular}




\begin{tabular}{|c|c|c|c|}
\hline 15 & $\begin{array}{l}L u=\frac{\tau^{2} h}{6}\left(\varepsilon-\frac{1}{2}\right)\left[\left(u^{3} u_{x x}+u^{2}\left(u_{x}\right)^{2}\right)_{x}+u\left(u^{2} u_{x}\right)_{x x}\right]_{x}+ \\
+\frac{\tau h^{2}}{12}\left[u^{2} u_{x x x}+3 u u_{x} u_{x x}+\left(u^{2} u_{x}\right)_{x x x}\right]_{x}+\frac{\tau^{3} a}{6}\left[u^{3} u_{x} u_{x x}+\right. \\
\left.+u^{2}\left(u_{x}\right)^{3}\right]_{x}+\frac{\tau^{3}}{9}\left[u u_{x}\left(u^{2} u_{x}\right)_{x}\right]_{x}-\frac{\tau^{3}}{24}\left(u^{4} u_{x}\right)_{x x x}-\frac{\omega h^{4}}{24 \tau} u_{x x x x x}\end{array}$ & да & нет \\
\hline 16 & $\left\{\begin{array}{l}L u=-\frac{\tau h^{2}}{48}\left(12 u u_{x} u_{x x}+5 u^{2} u_{x x x}+2\left(u_{x}\right)^{3}\right)_{x c}+\frac{\tau^{3}}{18}\left(4 u^{2}\left(u_{x}\right)^{3}+\right. \\
\left.+u^{3} u_{x} u_{x x}-u^{2}\left(u_{x}\right)^{3}\right)_{x}-\frac{3}{128} \frac{\pi^{4}}{\tau} \cdot u_{x x x x}-\frac{\tau^{3}}{24}\left(u^{4} u_{x}\right)_{x x x}\end{array}\right.$ & да & пгет \\
\hline 17 & $\begin{aligned} L u= & \frac{h^{2}}{12} u_{x} u_{x x}-\frac{25}{72} \tau^{2}\left[u^{2}\left(u_{x}\right)^{2}\right]_{x}-\frac{\tau^{2}}{6}\left[u\left(u^{2} u_{x}\right)_{x}\right]_{x}+ \\
& +\frac{\tau^{2}}{6}\left(u^{3} u_{x x}\right)_{x x}\end{aligned}$ & да & FICT \\
\hline 18 & $\begin{aligned} L u= & \frac{h^{4}}{180}\left[-120 x^{4} u\left(u_{x}\right)^{5}-300 x^{3} u^{3} u_{x}\left(u_{x x x}\right)^{2}-\right. \\
& -200 x^{4} u^{3}\left(u_{x}\right)^{2} u_{x x x}+u\left(1-x^{4} u^{4}\right) u_{x x x x x}- \\
& -600 x^{4} u^{2}\left(u_{x}\right)^{3} u_{x x}+10\left(1-5 x^{4} u^{4}\right) u_{x x} u_{x x x}+ \\
& \left.+5\left(1-5 x^{4} u^{4}\right) u_{x} u_{x x x x}\right]\end{aligned}$ & да & HET \\
\hline
\end{tabular}

Таблица 2

\begin{tabular}{|c|c|c|c|c|}
\hline \multirow{2}{*}{ Cxema } & \multirow{2}{*}{$\Pi$ П.форма п.д.п. } & \multicolumn{3}{|c|}{ Coollctвa } \\
\hline & & II & $M$ & $I$ \\
\hline $\begin{array}{l}\text { Ileiimarra- } \\
\text {-Pнхт- } \\
\text { мaitepa }\end{array}$ & $\begin{array}{l}u_{t}+\bar{p}_{x}=\left(z_{1} p_{x x}+z_{2} u_{x}^{2}\right)_{x} \\
v_{t}-u_{x}=-\left(z_{1} u_{x}\right)_{x x} \\
E_{\ell}^{\prime}+(u \bar{p})_{x}=\left[z_{1} u p_{x x}+p\left(z_{1} u_{x}\right)_{x}+2 z_{1} u_{x} p_{x}+z_{2} u u_{x}^{2}\right]_{x} \\
\text { плли } \\
\varepsilon_{t}+\bar{p} u_{x i}=z_{1} u_{x x} p_{x x}+z_{2} u_{x}^{3}+\left[p\left(z_{1} u_{x}\right)_{x}+2 z_{1} u_{x} p_{x}\right]_{x} \\
z_{1}=\frac{\tau^{2} \varrho^{2} c^{2}}{24}-\frac{h^{2}}{21}, z_{2}=\left(\varrho^{2} c^{2} p_{\varepsilon}+p^{2} p_{s \varepsilon}-2 p p_{\varepsilon v}+p_{\imath v}\right) \\
\bar{p}=p+\omega\end{array}$ & IreT & Нет & да \\
\hline $\begin{array}{l}\text { ЛІоліо- } \\
\text { стью нон- } \\
\text { серпатил- } \\
\text { ная По- } \\
\text { пова II } \\
\text { Самар- } \\
\text { стого }\end{array}$ & $\begin{array}{l}u_{l}+p_{x}=-\left(\frac{\tau}{2}-\alpha \tau\right)\left(\varrho^{2} c^{2} u_{x}\right)_{x} \\
v_{l}-u_{x}=0 \\
E_{t}+(u p)_{. c}=-\left(\frac{\tau}{2}-a \tau\right)\left(\varrho^{2} c^{2} u u_{x}\right)_{x} \\
\varepsilon_{t}+p u_{x}=-\left(\frac{\tau}{2}-a \tau\right) \varrho^{2} c^{2} u_{x}^{2} B U\end{array}$ & да & да & да \\
\hline
\end{tabular}




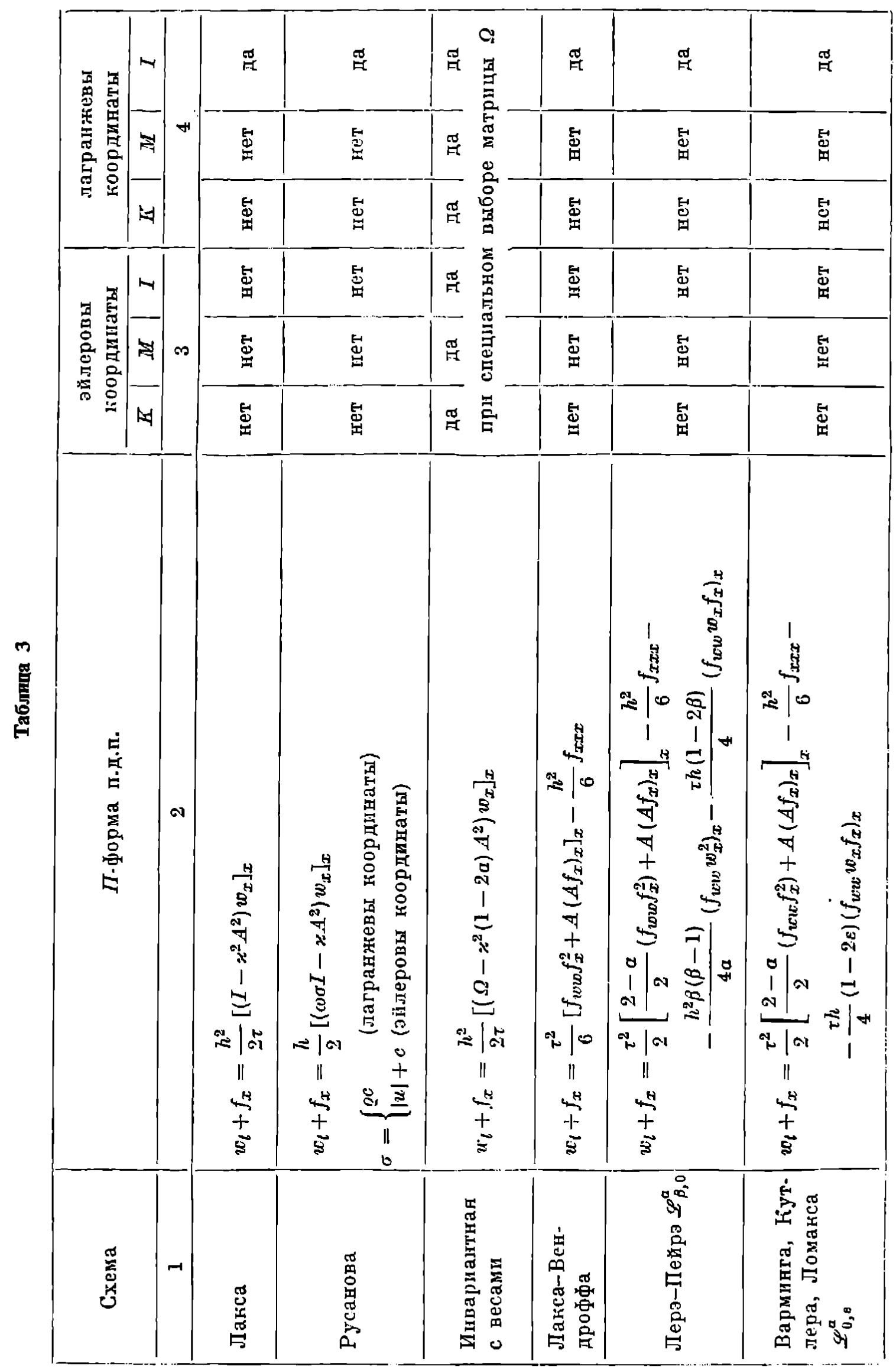




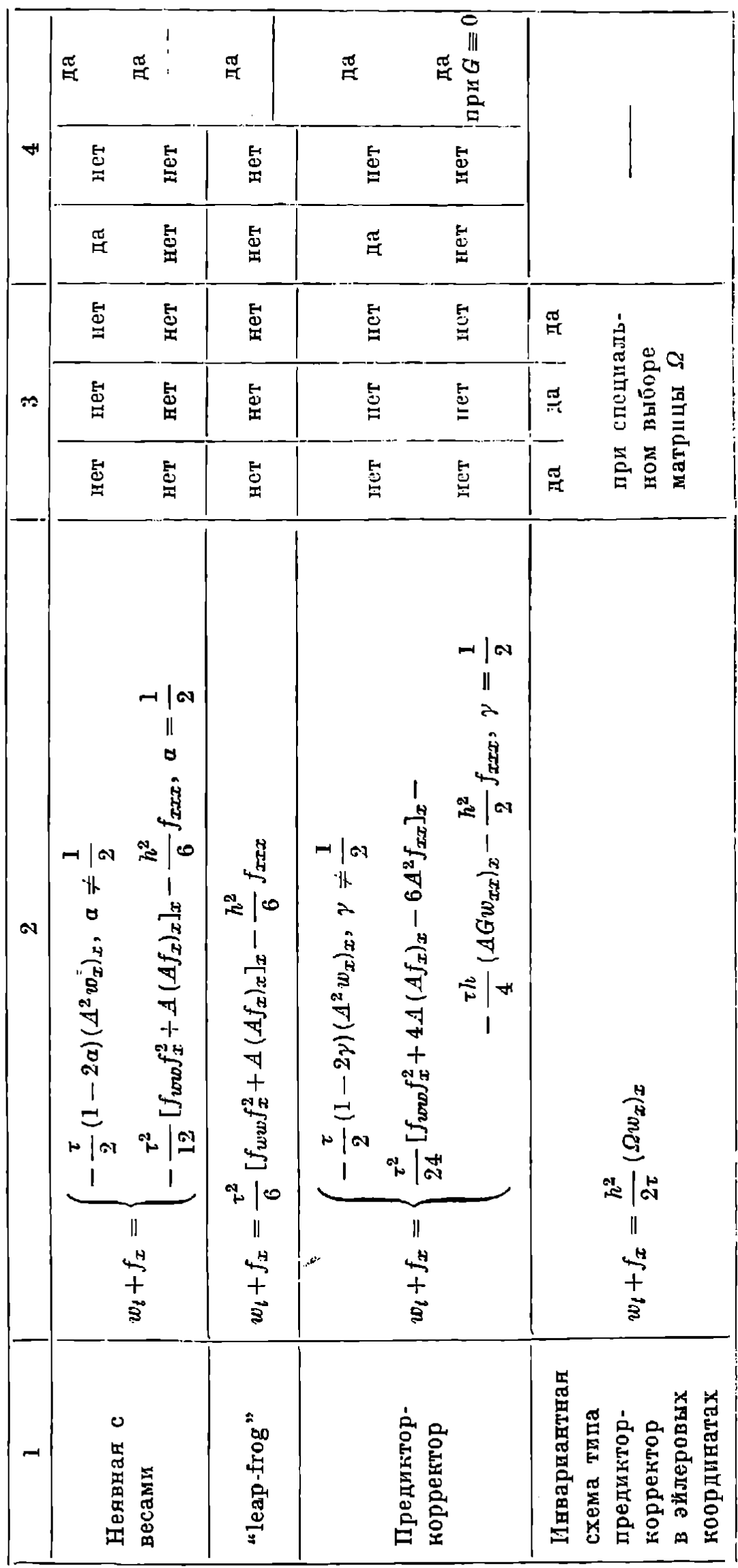


Построение разностных схем с заданными свойствами, огределенными в терминах п.д.п., а так определяются почти все осповные свойства разностных схем, сводится к построениго (в случае, папример, системы уравнениц одномерной газовой динамики) матрицы порядга $3 \times 3$, элементы которой должны удовлетворять ряду алгебраических соотпошений.

Действительно, возьмем для системы уравнений газовой динаМИкІ

$$
\frac{\partial w}{\partial t}+\frac{\partial f}{\partial x}=0
$$

разностнуг схему первого порядка аппрогсимации (для простоты явнуго)

$$
\begin{aligned}
& \quad \frac{w^{n+1}(x)-w^{n}(x)}{\tau}+\frac{f^{n}(x+h)-f^{n}(x-h)}{2 h}= \\
& =\left[\Omega\left(x+\frac{h}{2}\right) \frac{w^{n}(x+h)-w^{n}(x)}{h^{2}}-\Omega\left(x-\frac{h}{2}\right) \frac{w^{n}(x)-w^{n}(x-h)}{h^{2}}\right] .
\end{aligned}
$$

Здесь $\Omega$ - пога неизвестная $3 \times 3$ матрица, элементы ноторой ссть $O(\tau), w=(\varrho u, \varrho, \varrho E)^{\prime}, f=\left(\varrho u^{2}+p, \varrho u, \varrho u(E+p / \varrho)\right)^{\prime}, \imath-$ скорость, $v=1 / \varrho$ - удельный объем, $p=p(\varepsilon, \varrho)$ - давление, $\varepsilon$ - удельная внутренняя энсргия, $E=\varepsilon+u^{2} / 2$. П.д.ІІ. разностной схемы (10) имсет вид

$$
\frac{\partial w}{\partial t}+\frac{\partial f}{\partial x}=\frac{\partial}{\partial x}\left(C \frac{\partial w}{\partial x}\right)
$$

где $C=\Omega-\frac{\tau}{2} A^{2}, \Lambda=d f / d w$. Тогда, как показано в [1], разностнал схема (10) пригадлежит глассу $I$, если элементы матрицы $\Omega$ удовлетворяют ряду соотношении. Далсе, разностнан схема принадлсянит гілассу $I$, если $X C=0\left(X \Omega=-\frac{1}{2} \tau \iota^{2} X\right)$, где $X A=\imath X$ и т.д.

\section{Литература}

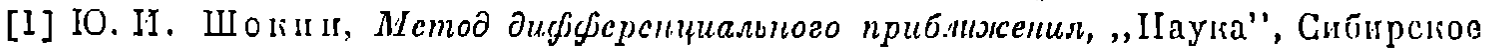
отдезсние, Новосио̆рсі木 1979, 224c.

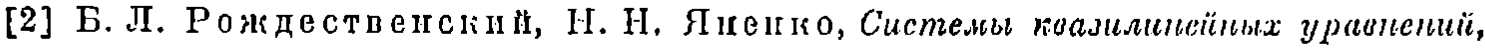
"Iayia", Mocina 1978, $088 \mathrm{c}$. 